Révisé, février 2008

\title{
Politique économique : avons-nous appris ?
}

\section{Jean Pisani-Ferry $\left({ }^{*}\right)$}

\footnotetext{
* Bruegel, Bruxelles, et Université Paris-Dauphine. Cet article est la version révisée de la conférence prononcée le 20 septembre 2007 au congrès de l'Association Française de Science Economique (AFSE) à l'issue de mon mandat de président de l'association. Il doit beaucoup à mes discussions avec Agnès Bénassy-Quéré, Benoît Coeuré et Pierre Jacquet et s'est enrichi des remarques critiques de Dominique Bureau, Paul Champsaur, JeanMichel Charpin, Benoît Coeuré, Michel Mougeot, Laurent Moulin et François Villeroy de Galhau. Je tiens enfin à remercier Jérémie Cohen-Setton pour son assistance diligente dans la préparation de ce papier.
} 


\title{
Résumé
}

Cet article examine ce que les développements contemporains de la recherche nous ont appris sur les politiques économiques et les leçons qui en ont été tirées dans la formulation et la mise en œuvre de la politique économique en France. Les avancées de la recherche sont rassemblées autour de trois directions principales: le caractère intertemporel des comportements et des politiques ; l'imperfection de l'information et ses implications pour les politiques économiques ; enfin les contraintes d'économie politique. Sont ensuite examinées trois grandes innovations par lesquelles les politiques économiques se sont approprié ces avancées : l'institution indépendante, la règle flexible et le contrat incitatif. La mise en œuvre de ces innovations en France est comparée à l'expérience internationale dans les domaines de la politique monétaire, de la politique budgétaire et de la gestion publique. Sont enfin explorées les raisons pour lesquelles la France accuse un retard dans l'appropriation et la mise en œuvre de ces innovations.

\begin{abstract}
This paper examines what economic policy has learned from the contemporary developments in economic research and which lessons have been drawn from them for the formulation and the implementation of French economic policy. Advances in research are grouped under three headings: the intertemporal character of private behaviour and public policies; imperfect information and its implications for policy; and political economy constraints. Next, the paper reviews three main innovations through which economic policy has adapted to this change in perspective: the independent institution; the flexible rule; and the incentive contract. The implementation of those innovations in France is compared to international experience in the fields of monetary policy, fiscal policy and public management. Finally, the paper discusses what France has been slow to adopt and implement those innovations.
\end{abstract}

JEL : B2, E6 
Pourquoi les performances économiques de la France sont-elles décevantes ? Cette question, qui est au cœur du débat politique, fait discussion parmi les économistes. Elle est généralement abordée sous un angle analytique, ce qui conduit à incriminer alternativement, ou conjointement, les politiques macroéconomiques conduites par les gouvernements successifs, le fonctionnement des marchés des produits, du travail ou des capitaux et l'impuissance à les réformer, ou encore le niveau et l'allocation de la dépense publique. L'objet de ce papier est d'examiner si la politique économique dans notre pays ne souffre pas aussi d'un défaut plus profond, s'il ne lui a pas systématiquement manqué de savoir tirer les leçons des développements de l'analyse économique pour en traduire les avancées en modernisant ses institutions, ses principes et ses procédures.

Dans une large partie du monde, les développements contemporains de la recherche ont en effet profondément transformé la vision et la pratique de la politique économique. Par vagues successives, une série de percées théoriques ou méthodologiques sont venues assaillir les fondements de l'édifice conceptuel qui avait semblé prendre forme stable au cours des années soixante. Comme souvent avec les révolutions, ces innovations se sont généralement présentées sous la figure de la radicalité, allant parfois jusqu'à mettre en cause l'utilité même de l'intervention publique. Assez rapidement, cependant, elles ont été intégrées au corpus des connaissances et ont, un peu partout, été prises en compte dans la définition et la mise en œuvre des politiques publiques par le canal d'innovations institutionnelles ou procédurales.

La France, cependant, semble en être bien souvent restée à des paradigmes, des institutions et des pratiques qui ignorent largement ces avancées. Quand bien même elle les a faites siennes dans tel ou tel domaine, c'est souvent à contrecœur, sous l'effet de la contrainte européenne ou sous l'influence de partenaires internationaux. De plus, elle a généralement négligé d'insérer les innovations qu'elle mettait en œuvre au sein d'une représentation cohérente des objectifs et des moyens de l'action publique. En est résulté une remarquable schizophrénie, une tendance récurrente à ne pas dire ce qu'on fait et à ne pas faire ce qu'on dit, dont trente ans de réflexion sur les conditions d'efficacité des politiques économiques nous ont pourtant appris combien elle était dommageable.

Ce retard d'apprentissage français est-il avéré, et s'il l'est, d'où vient-il ? Pour explorer cette question, je retrace d'abord ce que les développements contemporains de la recherche nous ont appris sur les politiques économiques (première partie). J'examine ensuite trois grandes innovations par lesquelles ces politiques se sont approprié ces avancées (deuxième partie). J'en viens ensuite à la politique économique française, dont je m’attache à évaluer la singularité en comparaison de l'évolution internationale en matière de politique monétaire, de finances publiques et de gestion publique (troisième partie). Je propose et discute enfin des hypothèses sur les raisons de cette singularité (quatrième partie).

\section{Trois leçons de la recherche}

Que savons-nous aujourd'hui de la politique économique que Valéry Giscard d'Estaing et Raymond Barre ne savaient pas ${ }^{1}$ ? L'idée que nous nous faisons aujourd'hui des conceptions des années soixante ou soixante-dix est sans doute assez caricaturale, et la naïveté que nous prêtons trop aisément aux décideurs des générations passées cadre mal avec ce que ne savons

\footnotetext{
${ }^{1}$ La question est empruntée à Blanchard (2000) qui, sous le titre "What Do We Know about Macroeconomics that Fisher and Wicksell Did Not?”, propose un panorama des avancées de la macroéconomie au vingtième siècle.
} 
de leur expérience ou de leur culture. Ce qui est vrai, cependant, c'est que la théorie des années soixante leur offrait une représentation remarquablement ingénue de la politique économique : toute décision semblait incomber à un monarque décrit par Jean-Jacques Laffont (2000) dans ses Clarendon lectures comme un parfait juge, un parfait décideur, et un parfait mandataire des volontés du peuple (ou si l'on préfère au dictateur omnipotent, omniscient, et bienveillant évoqué par Avinash Dixit (1996) dans ses Munich lectures).

Les trente dernières années ont apporté un renouvellement profond, que l'on peut organiser autour de trois thèmes qui ont structuré la recherche contemporaine : l'intertemporalité, l'information imparfaite, et l'économie politique ${ }^{2}$.

Ce changement de perspective a mis en pièces la vision traditionnelle. L'approche intertemporelle a rappelé que les monarques ne sont pas immortels, l'économie de l'information a montré qu'ils ne sont pas omniscients, et l'économie politique a suggéré qu'ils n'étaient pas tous constamment animés par le seul souci du bien public. Mais chacun de ces apports a, aussi, ouvert un champ nouveau à la politique économique: l'approche intertemporelle parce qu'elle suscite de nouveaux arbitrages, l'information imparfaite parce qu'elle met en cause la capacité du marché à atteindre l'optimum, et l'économie politique parce qu'elle complexifie le jeu avec les acteurs. Plutôt que comme une série de limites qui viendraient réduire le domaine de la politique économique, ces apports doivent être vus comme une série de défis auxquels il a fallu trouver de nouvelles solutions doctrinales, institutionnelles ou procédurales.

\subsection{Intertemporalité}

Que la politique économique ait un caractère intertemporel n'avait évidemment pas échappé à ses pères fondateurs. Mais cette dimension a pris dans une série de domaines une importance sans cesse croissance à partir des années soixante-dix. Cette évolution doit sans doute aussi bien aux développements théoriques de la deuxième moitié du XXème siècle que, plus concrètement, à l'expansion des marchés financiers, dont l'effet a été de détendre les contraintes de liquidité.

S'il faut choisir un point de départ, le meilleur est probablement la fameuse leçon de Friedman sur la verticalité de la courbe de Phillips à long terme (Friedman, 1968). Elle a longtemps été regardée comme le point de départ de la critique du keynésianisme, que les anticipations rationnelles allaient ensuite amplifier. Avec le recul, cependant, et la reformulation de l'approche keynésienne sur des fondements microéconomiques mieux assurés, la radicalité de la critique s’est émoussée, et les anticipations rationnelles ont ellesmêmes perdu de leur propriétés corrosives. Demeurent l'importance d'anticipations informées dans la détermination des comportements courants, et l'accent sur la sous-optimalité à long terme d'une politique guidée par l'optimisation à court terme, deux inflexions qui introduisent l'une et l'autre la dimension intertemporelle. C'est avec Kydland et Prescott (1977) qu'est apparue la pleine portée de cette nouvelle perspective pour la politique économique, avant que Barro et Gordon (1983) en donnent une version pédagogique appliquée à la politique monétaire.

\footnotetext{
${ }^{2}$ D’autres caractérisations seraient certainement possibles, qui mettraient davantage l'accent sur les démarches théoriques - par exemple sur les fondements microéconomiques de la macroéconomie - ou sur les méthodologies empiriques - par exemple sur le développement de l’économétrie sur données individuelles. La catégorisation retenue ici a l'avantage d'être utilisable à la fois pour ordonner les apports de l'analyse et pour réfléchir à la politique économique elle-même.
} 
Il est difficile de sous-estimer l'influence que ces travaux ont eus sur les doctrines monétaires ${ }^{3}$. En témoignent l'invocation obsessionnelle des notions de crédibilité et de réputation par les banquiers centraux d'Europe continentale (à tel point que l'existence d'arbitrages instantanés en vient parfois à être niée), mais aussi des réflexions sophistiquées comme celle de Mervyn King (2004), le gouverneur de la Banque d'Angleterre, qui définit le problème central du banquier central comme « l'incertitude sur les décisions futures résultant de ce qu'il n'est ni possible ni souhaitable de contraindre les décisions [de ses] successeurs » ${ }^{4}$.

L’influence de la démarche intertemporelle ne se limite cependant pas au champ monétaire. Dans deux domaines au moins de la politique macroéconomique, elle a sensiblement renouvelé les doctrines et, parfois aussi, les institutions de la politique économique. Il s'agit de l'équilibre extérieur d'une part, des finances publiques d'autre part".

Jusqu'au milieu des années quatre-vingt, la dimension intertemporelle de l'équilibre extérieur était une notion théorique pour la plupart des pays, et même parmi eux pour la plupart des pays industriels. En l'absence de liberté des mouvements de capitaux, le financement d'un déficit des paiements courants était sévèrement limité dans son montant et sa durée. La politique économique vivait sous l'emprise de la "contrainte extérieure ». La libéralisation très rapide des comptes financiers (Graphique 1) a profondément changé le paysage et suscité une réflexion renouvelée sur la dimension intertemporelle de la balance des paiements (Obstfeld et Rogoff, 1996). Comme l’ont illustré les débats récents sur les déséquilibres globaux de balance des paiements, l'approche normative de l'équilibre extérieur s'en est trouvée profondément perturbée : on a pu par exemple soutenir que le déficit américain était optimal (Engel et Rogers, 2006) ; il en va de même des pays européens en situation de déficit prononcé comme l'Espagne, la Grèce ou les Pays Baltes, pour lesquels l'analyse peine à produire une prescription (Blanchard, 2007).

\section{Graphique 1 : Ouverture financière des pays du G7, 1970-2004}

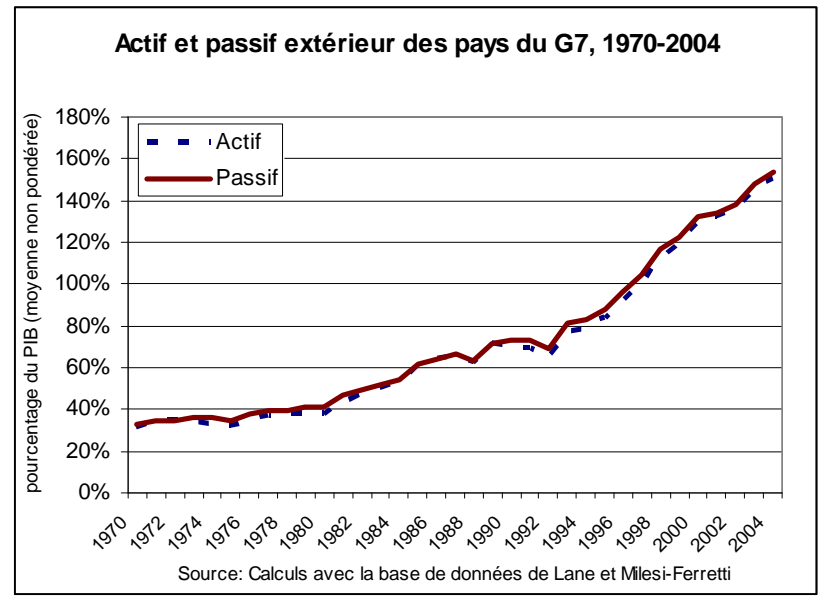

\footnotetext{
${ }^{3}$ Parce qu'il a fourni une représentation extrêmement simple et aisément communicable du problème de la crédibilité, l’article de Barro et Gordon (1983) a notamment eu une influence considérable sur l'évolution des doctrines et des institutions monétaires.

${ }^{4}$ L’impossibilité résulte selon King du caractère collectif des décisions. La non-désirabilité de l'ignorance quant aux états de nature futurs.

${ }^{5}$ On pourrait ajouter la dimension environnementale.
} 
En matière de finances publiques, les considérations intertemporelles ne datent pas d'hier. La crainte du défaut a certainement préexisté à toute réflexion sur les vertus conjoncturelles des déficits, Ricardo a précédé Barro, et Necker a énoncé deux siècles avant Sargent et Wallace les rudiments de leur arithmétique déplaisante. Cependant l'évolution récente est marquée par la montée des préoccupations relatives à la soutenabilité. Il y a certainement d'abord des raisons très concrètes à cette évolution : la dégradation des ratios d'endettement dans les pays avancés depuis les années quatre-vingt; l'inquiétude quant à l'avenir consécutive au vieillissement; et l'élimination de l'option d'une monétisation inflationniste à la suite de l'indépendance de la banque centrale ou du fait de l'adhésion à une union monétaire. La littérature économique y a aussi contribué en mettant au point, avec les modèles à générations imbriquées et la comptabilité générationnelle, les outils d’une approche rigoureuse.

Au total, la prise en compte de la dimension intertemporelle a conduit à allonger l'horizon et à prendre mieux en compte le jeu entre décision publique et anticipations privées, bref à ne plus examiner les choix publics à travers le seul prisme des arbitrages instantanés.

\subsection{Economie de l'information}

Dans un article rétrospectif publié il y a quelques années, Joseph Stiglitz (2000) soutient que l'économie de l'information a été la principale innovation théorique du XXème siècle. Il y souligne qu'au contraire d'autres développements que les écrits antérieurs portaient en germe, les conséquences de l'imperfection de l'information - un fait qui, en lui-même, était bien entendu reconnu - ont été ignorées jusqu'aux années soixante et soixante-dix. C'est à partir de ce moment que les travaux de Vickrey (1961) sur les enchères, d'Arrow (1963) sur l'alea moral, d'Akerlof (1970) sur le comportement du consommateur en situation d'information incomplète, de Mirlees (1971) sur la taxation optimale ou d'Hurwicz (1973) sur la compatibilité incitative ont initié un traitement rigoureux de la question, avant bien sûr que Stiglitz lui-même montre la puissance de l’hypothèse d'asymétrie d’information.

Même en faisant la part du plaidoyer pro domo, il n'est guère douteux que le traitement systématique de la question a été à l'origine de renouvellements profonds dans de nombreux domaines. Pour s'en tenir à la politique économique, celle-ci s'est trouvée à la fois confortée dans son utilité et mise en cause dans ses fondements. En montrant que les résultats d'ArrowDebreu n'étaient vrai que sous des hypothèses extrêmement exigeantes quant à la disponibilité et à la distribution de l'information, et plus concrètement en mettant en évidence comment l'asymétrie d'information pouvait causer chômage involontaire, rationnement du crédit ou inefficiences sur les marchés financiers, Stiglitz et ses co-auteurs ont rouvert à la politique économique des espaces qu'une vision absolutiste de l'équilibre de marché avait voulu fermer.

Cependant la même démarche a, bien évidemment, abouti à souligner la naïveté des représentations usuelles de la décision publique et les risques d'interventions inefficaces ou contre-productives lorsque l'information du décideur est imparfaite. En mettant l'accent sur ces contraintes informationnelles, elle a conduit à repenser profondément l'économie publique, allant souvent jusqu'à en changer radicalement les termes. Les notions de compatibilité incitative, d'alea moral ou de sélection adverse sont devenues centrales dans toutes les réflexions sur les modalités de l'intervention publique en situation d'information imparfaite quant aux caractéristiques ou aux comportements des agents privés. 
La reconnaissance du caractère imparfait de l'information affecte donc profondément la conception de la politique économique. Au niveau le plus général elle conduit, comme le développe Dixit (1996), à tenir marchés et gouvernements comme des institutions l'une et l'autre imparfaites et l'une et l'autre nécessaires. La question posée au décideur public n'est plus seulement de savoir si son intervention est justifiée, elle n'est plus seulement de savoir quelle est la bonne politique, elle est de fixer quels sont les systèmes de décision et d’intervention susceptibles de faire le meilleur usage de l’information limitée dont il dispose.

De manière plus directe, la révolution intellectuelle introduite par l'information imparfaite a transformé les fondements de l'économie normative, qui retenait sans discussion l'hypothèse d'une parfaite information du décideur. Elle a conduit à repenser les fondements de la gestion publique mais aussi la régulation des marchés, la supervision financière, ou les politiques sociales. Il est difficile de surestimer l’ampleur de cette révolution intellectuelle.

\subsection{Economie politique}

Le troisième facteur de changement a été la nouvelle économie politique, ou si l'on préfère l'approche positive de la politique économique qui s'est considérablement développée depuis les années quatre-vingt en faisant fond sur des travaux initialement disjoints, mais qui tous faisaient figure de provocations - lorsque Downs (1957) développait sa théorie économique de la démocratie, que Nordhaus (1975) cherchait à mettre en évidence un cycle des affaires électoral, ou que Buchanan (1975) écrivait qu'il « n’existe pas de personne, de décideur, qui maximise pour l'économie » et que « ce qui résulte [du processus de décision] est ce qui en résulte, un point c'est tout ».

Cette grille de lecture a d'abord accueillie avec réserves, particulièrement en France où les économistes ont coutume de se voir comme des conseillers au service d'un prince bienveillant (elle a été beaucoup plus facilement accueillie en Allemagne où ils se voient comme les avocats de la rationalité dans un monde travaillé par la politique). Elle a néanmoins fait peu à peu sa place en mettant en lumière les défaillances potentielles ou réelles de la décision politique. Dans ses versions naïves elle s’applique sans doute davantage aux Etats-Unis, où le financement direct des partis et des candidats par des groupes d'intérêt est chose admise, qu'en Europe où il est souvent banni. Mais l'économie politique ne se réduit évidemment pas à cette représentation. Comme l’indiquent Persson et Tabellini (1999), la décision politique est sujette à trois types de carences: la vulnérabilité des gouvernements démocratiques aux problèmes d'incohérence temporelle, l'électoralisme, et l'orientation partisane. Aucun de ces dangers n’est absent en Europe, ni en France.

A partir des années quatre-vingt, la recherche a appliqué avec succès l'approche par l'économie politique à la politique monétaire, à la politique budgétaire, ou aux politiques de régulation. Elle a notamment explicité les raisons pour lesquelles certains pays pouvaient conduire de manière obstinée des politiques budgétaires manifestement vouées à l'impasse Alesina et Drazen, 1991) ou des politiques de l'emploi résolument inefficaces (Saint-Paul, 1993) et a mis en lumière, quant à l'incidence des développements politiques sur les résultats économiques, des faits stylisés assez nets pour mettre en question la séparation traditionnelle entre analyse politique et analyse économique ${ }^{6}$.

\footnotetext{
${ }^{6}$ Persson et Tabellini (1999) donnent un survey détaillé de la littérature théorique et empirique sur l’apport de l'économie politique dans le domaine macroéconomique.
} 
L'économie politique ne rend pas la politique économique nuisible, comme l'incohérence temporelle ne la rendait pas sans objet. Mais elle attire l'attention sur ses risques de défaillance et invite à réfléchir aux conditions de son efficacité. S'il est permis de critiquer la représentation que certains modèles donnent des motivations des dirigeants politiques, il n'est plus possible d'ignorer les contraintes d'économie politique auxquelles ceux-ci sont soumis.

\subsection{Synthèse}

Ce qui justifie de réunir les trois percées ici mises en exergue est qu'elles ont toutes trois affecté, non les objectifs, mais la représentation de la politique économique et de sa relation à l'économie privée. Elles ont en outre une forte cohérence intellectuelle, non seulement en ce qu'elles identifient chacune un type de contrainte qu'une décision publique doit prendre en compte, mais aussi parce que ces contraintes peuvent se cumuler et se combiner. Ce n'est pas par hasard si nombre de modèles combinent asymétries d'information et contraintes d'économie politique dans un cadre intertemporel ${ }^{7}$. Soumettre une proposition de politique économique au triple test de la dimension intertemporelle, du traitement implicite de l'information et de l'économie politique est ainsi devenu un exercice indispensable.

\section{Trois innovations en politique économique}

C’est seulement graduellement qu'ont été inventées et mises en place des réponses aux défis issus des développements de la théorie économique. En ont résulté une série d’innovations doctrinales ou institutionnelles qui ont substantiellement transformé la manière de concevoir et de conduire la politique économique. Ces innovations dans l'art de gouverner sont généralement apparues dans tel ou tel domaine particulier. Mais elles ont souvent eu une portée plus générale et se sont plus ou moins largement diffusées dans d'autre domaines : il n’est pas excessif de dire qu'elles ont largement eu le caractère de technologies génériques.

\subsection{La délégation}

La première innovation a été la délégation de responsabilité à une institution indépendante. Son origine est bien antérieure aux années soixante-dix puisque sans même remonter à la création de la Bank of England en 1694, on peut citer celles de l'Interstate Commerce Commission en 1887, de la Securities and Echange Commission en 1934, et de la Bundesbank en 1947. Mais c'est dans le dernier quart du XX ${ }^{\text {eme }}$ siècle que le modèle s'est généralisé, avec en particulier l'indépendance des banques centrales, l'autonomisation des autorités de la concurrence et la création de nombreuses autorités sectorielles de régulation (Cohen, 2001).

La délégation à une institution indépendante vise à remédier à la fois au court-termisme et à la politisation des décisions. Même lorsque le mandat de l'institution est défini avec précision par le législateur et que son responsable est soumis à l'obligation de rendre des comptes, le défaut de cette solution est évidemment de protéger celui-ci de la sanction démocratique. La recherche s'est attachée à déterminer quand les avantages l'emportent sur l'inconvénient .

Dans la modélisation qu'en donnent Maskin et Tirole (2004), les électeurs sont imparfaitement informés quant à la politique qui maximise le bien-être social (par exemple, ils ne savent pas s'il est préférable de lutter pour la croissance ou contre l'inflation) et ont une

\footnotetext{
${ }^{7}$ C'est le cas par exemple du modèle de Maskin et Tirole (2004) sur le choix entre confier la conduite d'une politique économique à des élus ou à des magistrats.
} 
certaine probabilité de le découvrir, mais seulement avec retard. Ils ont le choix entre s'en remettre à des élus, au risque que ceux-ci privilégient, non la bonne décision, mais celle qui maximise leurs chances de réélection; ou bien à des technocrates, au risque que ceux-ci privilégient, de manière doctrinaire, la politique qui a leur faveur. Le problème est donc l'écart éventuel entre les préférences de l'agent à qui la décision est déléguée et les préférences sociales. Maskin et Tirole montrent que la solution technocratique est préférable à la gouvernance politique lorsque l'électorat a une faible probabilité de découvrir quelle était la bonne décision - en d'autres termes quand la matière est très complexe ou technique.

Dans la modélisation d'Alesina et Tabellini (2007), les problèmes viennent plutôt de l'incertitude sur les qualités de l'agent et de l'inobservabilité de son effort. Sa motivation est la reconnaissance de son talent (dont l'observation est bruitée par l'effort et les alea) tandis que pour l'élu, elle est d'être réélu (ce qui dépend du résultat obtenu et donc, outre son talent et de son effort, d'événements aléatoires). Eux aussi concluent que le choix dépend de la technicité de la matière, et aussi des effets distributifs de la décision.

Ces deux formalisations traduisent bien comment information imparfaite et contrainte d'économie politique peuvent se combiner dans un cadre intertemporel, et pourquoi la délégation ne peut réellement s'appliquer qu'à des domaines particuliers dans lesquels les électeurs ne savent pas évaluer les performances des élus, et ont en revanche des préférences suffisamment stables pour qu'il soit possible de définir ex ante le mandat d'une agence indépendante (comme l'observent Alesina et Tabellini, personne ne songe à confier les Affaires étrangères à une telle agence). D'autres critères peuvent être retenus (existence d’arbitrages, implications distributives des décisions, risque d'incohérence temporelle).

\subsection{La règle flexible}

La seconde innovation a été de contraindre la capacité discrétionnaire du décideur politique par la fixation ex ante d'une règle de comportement. Apparue sous impulsion monétariste dans les débats des années soixante et soixante-dix avec la proposition de norme de croissance annuelle de la masse monétaire, puis théorisée par Kydland et Prescott (1977), la méthode se veut d'abord une réponse au problème d'incohérence temporelle auquel peuvent être sujets des gouvernements soucieux du bien public, mais dont l'horizon de décision est trop court.

En matière monétaire, il a semblé un temps, malgré les efforts de certains théoriciens et l'attachement aux agrégats professé par la Bundesbank, que la notion de règle ne survivrait pas aux déboires qu'ont connu les stratégies monétaristes dans les années soixante-dix et quatre-vingt. De fait, elle n’y a guère survécu aux Etats-Unis ${ }^{8}$. Mais elle a fait fortune en Europe et dans le reste du monde.

En matière monétaire, la renaissance est venue de Nouvelle-Zélande avec l'invention en 1990 de l'inflation targeting, que l'on peut voir comme une forme de règle monétaire sans monnaie. Le modèle s'est rapidement diffusé, d'abord dans les pays industriels (Canada, Royaume-Uni, Suède..) puis dans un nombre croissant de pays émergents (Mexique, Brésil, Afrique du Sud..) à l'occasion de leur passage de stratégies de change fixe à des stratégies de changes flottants. Il constitue une référence obligée pour les réflexions de stratégies monétaires, même celle des banques centrales qui conservent des réticences à son égard.

\footnotetext{
${ }^{8}$ Ben Bernanke est cependant connu comme un avocat de l'inflation targeting, et tente d'orienter graduellement la Réserve fédérale vers un cadre de politique monétaire moins discrétionnaire que celui qu'il a hérité d’Alan Greenspan.
} 
A l'occasion cette renaissance, le modèle s'est surtout considérablement sophistiqué, sur deux plans : d'abord en faisant explicitement place à la stabilisation de l'output avec ce qu'il est convenu d'appeler le ciblage flexible (flexible inflation targeting); ensuite en prenant une dimension explicitement intertemporelle avec les versions avancées de l'inflation targeting mises en place en Norvège ou en Suède, dans lesquelles la banque centrale s'attache à guider les anticipations en publiant une prévision d'inflation conditionnelle à une trajectoire explicite pour ses propres taux d'intérêt (Svensson, 2007). Les modèles avancés d'aujourd'hui n'ont ainsi plus grand-chose à voir avec la vision mécaniste d'une norme de croissance monétaire.

En matière budgétaire, la réflexion sur les règles a été plus tardive et moins rigoureuse (Kopits et Symansky, 1998). Les justifications données pour y recourir se bornent souvent à un recensement des facteurs, souvent d'ordre politique, qui concourent au biais vers le déficit, sans que soit précisément analysé le coût potentiel d'un renoncement à la discrétion. Les premières règles ont, aussi, généralement eu un caractère grossier (avec, notamment, les règles d'équilibre, ou la "règle d'or » allemande qui n’autorise le déficit qu'en contrepartie d'un investissement), avant de se raffiner en prenant en compte le cycle (objectifs exprimés en terme de solde structurel), en retenant une cible de dette qui laisse une grande marge de manœuvre dans le court terme, ou en fixant une norme de dépense qui laisse le déficit fluctuer en fonction des ressources ${ }^{9}$. L'évolution a donc été comme en matière monétaire en direction de règles flexibles qui laissent place à une importante marge de manœuvre discrétionnaire, mais fixent des principes et des bornes.

La pratique des règles comme les réflexions des théoriciens se sont donc écartées de la vision par trop mécaniste des premiers temps pour faire place à ce qu'il est convenu d'appeler la discrétion contrainte (Bernanke, 2003). Le décideur - banque centrale ou autorité budgétaire conserve une latitude importante dans l'interprétation des données, la gestion du risque et la prise en compte d'autres objectifs, mais est soumis à une discipline intellectuelle qui donne cohérence à ses actions et sert de garde-fou contre l'opportunisme ou l'éclectisme sans cohérence. C’est ce qui permet de parler de règle flexible.

\subsection{Le contrat incitatif}

La troisième innovation, le contrat incitatif, s'inscrit dans une tradition ancienne que Laffont et Martimort (2002) font remonter à l'analyse du contrat de métayage par Adam Smith. Mais c'est à partir des années quatre-vingt, dans le contexte de la dérégulation des utilities initiée aux Etats-Unis, que, dans la foulée des travaux sur le mechanism design ${ }^{10}$, s'est structurée la réflexion sur le modèle principal-agent et les propriétés souhaitable du contrat qui les lie en situation d'information imparfaite (Baron et Myerson, 1982, Laffont et Tirole, 1986). L'idée de base est que le contrat doit être spécifié de telle sorte que l'agent ait intérêt à révéler l’information privée dont il dispose et qui lui donne un avantage stratégique sur le principal.

Cette démarche intellectuelle va à l'encontre d'une tradition ancienne, et solidement établie en France, selon laquelle le problème de la gestion publique est de spécifier de manière aussi précise que possible les objectifs et de prévoir de manière quasi-exhaustive l'ensemble des cas qui peuvent se présenter, afin de ne laisser aucune place à l’improvisation.

\footnotetext{
${ }^{9}$ Wyplosz (2005) fournit un bref survey des règles en vigueur dans le monde.

${ }^{10}$ Ces travaux viennent d'être couronnés par le prix Nobel, attribué conjointement à Hruwicz, Maskin et Myerson.
} 
La portée de l'approche par le contrat incitatif a rapidement dépassé le seul domaine des relations entre Etat et entreprise délégataire d'un service public. Elle a d'abord fourni un cadre intellectuel unifié pour penser les problèmes de délégation, qu'ils touchent aux services publics, aux partenariats public-privé, et aux relations entre l'Etat et ses démembrements en charge de régulations sectorielles ou spécifiques. Elle a ensuite été étendue à l'organisation interne des administrations (Tirole, 1994 ; Laffont, 2000) ; ou encore à la gouvernance des institutions internationales (Tirole, 2002).

Evidemment, chacun de ces problèmes a des dimensions spécifiques et il serait abusif de prétendre que l'organisation interne des services administratifs repose sur les mêmes solutions que la relation entre Etat et entreprises privées sous contrat. Demeurent, cependant, la similarité des problèmes d'asymétrie d'information et d'incitation et l'unité du cadre intellectuel apporté par les modèles de type principal-agent. Ce cadre est aujourd'hui couramment utilisé en politique économique dans des domaines très divers, non seulement dans la définition des contrats avec les agents privés ou la spécification des enchères, mais aussi dans l'organisation des relations entre les Etats et leurs agences ou dans l'organisation interne des services publics. Sans aller jusqu'à rémunérer le gouverneur de la banque centrale sur la base de ses performances macroéconomiques comme l'a suggéré Walsh (1995), des contrats incitatifs sont par exemple utilisés par plusieurs pays pour déterminer l'allocation des fonds publics entre des entités décentralisées (universités, écoles, services administratifs..).

\section{Les politiques économiques à l'épreuve des innovations}

La diffusion de ces innovations dans les différents champs de la politique économique a procédé inégalement d'un pays à l'autre et d'un domaine à l'autre. C'est en matière monétaire que la convergence des institutions et des pratiques est la plus forte. Le domaine budgétaire est marqué par une plus grande hétérogénéité. Quant à la gestion publique, elle reste très disparate, même entre les pays industriels.

\subsection{Politique monétaire}

\section{L'expérience internationale}

Directement mises en cause dans les années soixante-dix pour avoir, par naïveté, par myopie ou par faiblesse, exploité des arbitrages de court terme au détriment du long terme, les politiques monétaires ont été profondément transformées par la généralisation, dans le monde entier, du modèle de la banque centrale indépendante dédiée à un objectif prioritaire de lutte contre l'inflation (Graphique 2).

Le succès remporté contre l'inflation est l'un des plus spectaculaires qui soit. En 1980, seuls $10 \%$ des pays du monde enregistraient une inflation inférieure à 5\% On en comptait plus de $50 \%$ en 2006 et un seul pays, le Zimbabwe, est en situation d'hyperinflation. Même en faisant la part de la conjoncture, et d'autres facteurs structurels comme l'accroissement de la pression concurrentielle dans un contexte de mondialisation, le résultat est suffisamment net pour que soit validé, pour de nombreuses années au moins, le modèle institutionnel de l'institution monétaire indépendante. 


\section{Graphique 2 : Indépendance des banques centrales, années 80 et années 2000}

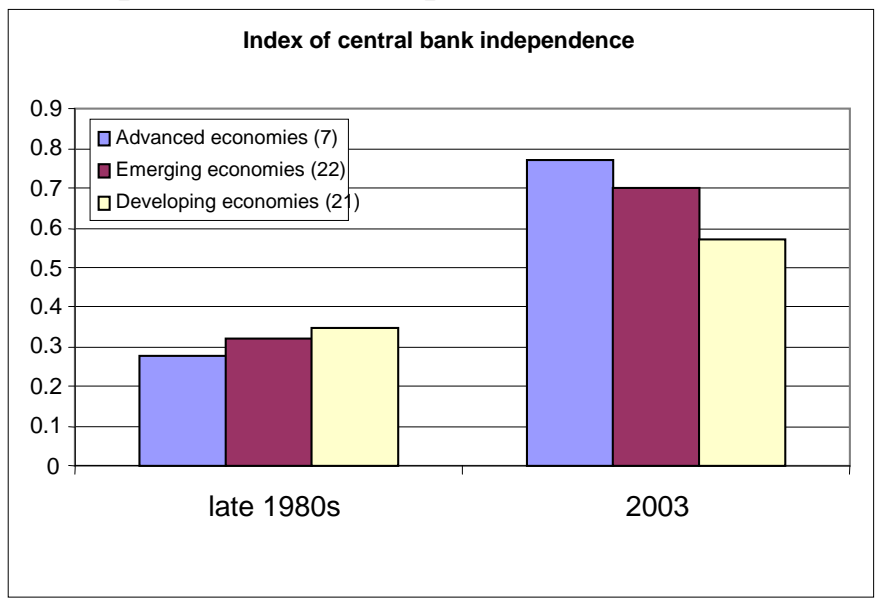

Source : Bénassy-Quéré et al. (2008) d'après Arnone et al. (2007). Un indice plus élevé correspond à une augmentation de l'indépendance.

Le modèle offre peu d'exceptions quant au choix des objectifs (celle des Etats-Unis mérite d’être notée, où le mandat de la banque centrale place sur le même plan stabilité des prix et stabilisation de l'output) mais de subtiles variations sur deux points :

- les termes du contrat par lequel le gouvernement ou le parlement délègue la responsabilité de la politique monétaire à une institution autonome. Entre la délégation inconditionnelle sur la base d'une formulation générale du mandat fixé à la banque centrale (ce qu'il est convenu d'appeler indépendance quant à l'objectif) et la simple autonomie dans la poursuite d'un objectif fixé par le gouvernement (indépendance instrumentale), il y a place pour une gradation ;

- la formulation de la stratégie monétaire, c'est-à-dire la manière dont la banque centrale indique par avance aux agents économiques comment elle corrigera des déviations de trajectoire et répondra à des chocs. Les différences portent ici sur la définition de l'objectif et sur le caractère plus ou moins explicite de la fonction de réaction de la banque centrale.

Sur ces deux points, il y a aujourd'hui concurrence pour la recherche du modèle d'avenir, sans que l'expérience acquise permette de trancher entre les visions alternatives. Pour être subtil et technique, le débat n'en est pas moins vif. Il oppose ceux pour qui la garantie du succès de la banque centrale repose sur le caractère absolu de son indépendance, et sa capacité à répondre au mieux aux chocs, sans que des règles préétablies contraignent son action ; et ceux pour lesquels une institution publique n'a pas légitimité pour définir son propre objectif, et qui voient dans la définition ex ante d'une stratégie une garantie tant d'efficacité que d'accountability. La Banque centrale européenne, qui a fixé elle-même sa définition de la stabilité des prix et se refuse à adopter une stratégie explicite d'inflation targeting, symbolise la première attitude, tandis que la Banque d'Angleterre, dont l'objectif est fixé par le gouvernement et dont la stratégie repose sur cet inflation targeting, incarne la seconde.

\section{Le cas français}

Avant qu'elle se fonde, en 1999, dans la zone euro, la politique française ne s'est jamais distinguée par une approche innovante de la politique monétaire. La Banque de France a été tenue sous tutelle le plus longtemps possible. Lors du débat sur la ratification du traité de Maastricht, François Mitterrand a, dans une réponse fameuse, nié la réalité du changement en 
indiquant que «les techniciens [seraient] chargés d'appliquer dans le domaine monétaire les décisions prises par les douze chefs d'Etat ou de gouvernement " ${ }^{11}$. Lorsque l'indépendance a été accordée à l'institution monétaire, en 1993, ce fut par un contrat de délégation - il est vrai à durée déterminée - d'une remarquable absence de sophistication. Sur le plan externe, l'indépendance de la BCE a été traitée dans la négociation comme une concession politique faite à l'Allemagne dont il convenait d'obtenir des contreparties, non comme le résultat d'une recherche conjointe en vue d'aboutir au meilleur modèle. Enfin les négociateurs français ont tenté de contenir l'étendue de la délégation en plaidant pour une politique de change indépendante de la politique monétaire, et gérée par les ministères des Finances plutôt que les banques centrales (Dyson et Featherstone, 1999, Pisani-Ferry, 2006, Henning, 2007).

Par l'effet de cette attitude constante, très peu d'efforts ont été consacrés à acclimater au système institutionnel et politique l'innovation majeure qu'a constitué l'indépendance de la banque centrale. Toute autre a été la démarche du Royaume-Uni, où la soumission de l'institution monétaire était pourtant solidement ancrée: en 1997, l'indépendance de la Banque d'Angleterre s'est accompagnée de la définition d'un contrat élaboré au gré duquel la celle-ci dispose d'une pleine indépendance opérationnelle mais ne fixe pas elle-même son objectif, et doit rendre des comptes si elle s'en écarte (ce qui est arrivé en 2007). Loin d'être le canal d'un assujettissement, le fait de laisser au chancelier de l'Echiquier la responsabilité de définir l’objectif de stabilité des prix est, en réalité, une manière subtile de lier les mains du politique, tout en manifestant symboliquement que la diffraction de l'autorité n'entame pas la cohérence de la politique économique (Bhundia et O’Donnell, 2002).

L'exemple britannique n'est évidemment pas transposable puisque la non-appartenance à la monnaie européenne offre à ce pays des marges de manœuvre dont la France ne dispose pas. Les contraintes de l'Union économique et monétaire n'interdisaient cependant pas hier de conduire une réflexion sur l'insertion de l'indépendance de la banque centrale dans le système de politique économique national, elles n'interdisent pas aujourd'hui d'imaginer, dans le respect des traités, des évolutions possibles dans le système de politique économique de la zone euro (Pisani-Ferry et al., 2008). Faute sans doute d'une telle réflexion sur les voies d'acclimatation d'un modèle allogène à la tradition politique nationale, l'adhésion au principe de l'indépendance de la banque centrale est restée remarquablement superficielle. En témoigne, au-delà de critiques à l'égard de la politique de la BCE qui peuvent être légitimes, la récurrence, de la part de personnalités politiques de premier plan appartenant à un spectre politique large, de déclarations mettant en cause soit le modèle institutionnel, soit l'objectif de la politique monétaire européenne. La persistance d'un hiatus important entre les conceptions françaises de politique monétaire et la réalité européenne pourrait finir par saper les bases de la participation du pays à la zone euro.

\subsection{Politique budgétaire et finances publiques}

\section{L’expérience internationale}

Comme la politique monétaire, la politique budgétaire telle qu'elle était envisagée (sinon toujours pratiquée) dans les années soixante-dix a subi la critique. Il lui a été reproché de privilégier la stabilisation à court terme au détriment de la soutenabilité à long terme, de se fonder souvent sur des informations inexactes, et de répondre à des motivations politiciennes

\footnotetext{
${ }^{11}$ Interview télévisée du 3 septembre 1992 à l’occasion du referendum sur le traité de Maastricht.
} 
étrangères au bien-être social. De fait, la montée des ratios de dette publique à partir du milieu des années soixante-dix a témoigné d’une perte de repères des politiques budgétaires.

La principale réponse donnée à ces préoccupations n’a pas été, comme en matière monétaire, la délégation à une institution indépendante. Cette solution a été proposée, notamment par Blinder (1997) et Wyplosz (2005), mais sa mise en œuvre impliquerait de pouvoir délimiter, au sein de la politique budgétaire, les aspects intertemporels, qui pourraient être confiés à un comité indépendant, des aspects allocatifs ou redistributifs, qui relèvent nécessairement du parlement. Aucun pays n’a jusqu'ici entrepris de tracer cette frontière (même si certains, comme la Belgique et les Pays-Bas, ont confié la préparation des hypothèses économiques sous-jacentes aux lois de finances à des offices publics indépendants).

La recherche de solutions s'est plutôt orientée vers la définition de règles de politique budgétaire qui laissent un certain degré de souplesse conjoncturelle et autorisent des décisions discrétionnaires, mais inscrivent les choix budgétaires dans une discipline intertemporelle. C'est le cas de beaucoup de pays en Europe, qu'ils soient ou non assujettis au Pacte de stabilité. Un récent recensement par la Commission européenne (2007) montre que la tendance a été constante depuis le début des années quatre-vingt dix (Graphique 3). Hors d'Europe, la tendance est moins nette, ne serait-ce qu'en raison de l'absence de toute contrainte à une gestion discrétionnaire du budget fédéral américain. Mais tous les Etats américains sauf un (le Vermont) ont adopté des règles budgétaires et que plusieurs pays industriels (Canada, Nouvelle-Zélande) ou émergents (Brésil) ont fait de même.

\section{Graphique 3 : Couverture des règles budgétaires nationales en Europe, 1990-2005}

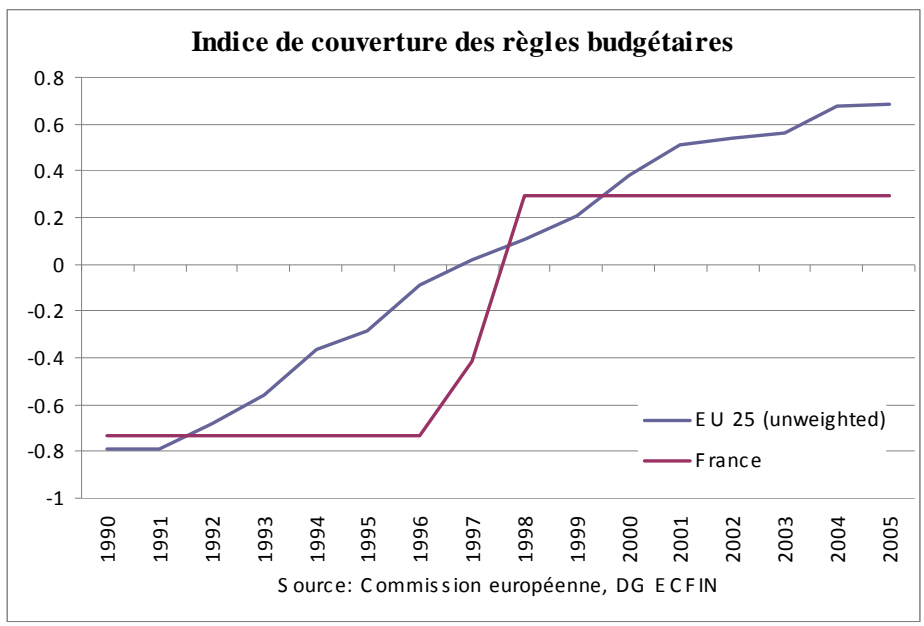

Source : Commission européenne. L’indice donne pour chaque pays une mesure de la couverture des finances publiques par des règles budgétaires. L’indice agrégé est la moyenne non pondérée des indices nationaux pour les pays de l’UE.

L'expérience britannique est ici aussi intéressante, en ce qu'elle vise à combiner cadre de moyen terme et flexibilité de court terme. Le « code de responsabilité budgétaire » de 1997 associe en effet une règle de déficit (la règle d'or selon laquelle le déficit ne peut pas excéder l'investissement public) et un objectif de dette (maintenir la dette publique au niveau de $40 \%$ du PIB) qui s'appliquent l'une et l'autre sur le cycle économique, et non année par année. Même si cette règle n'est pas sans défaut (elle est manipulable car elle repose sur un jugement 
quant à la longueur du cycle), il s'agit typiquement d'une mise en application de la philosophie de la discrétion contrainte.

La permanence des controverses autour du Pacte de stabilité indique que la gouvernance par les règles est loin de faire consensus parmi les économistes. Elle est certainement beaucoup plus discutée que l'indépendance des banques centrales. Pour autant, la tendance internationale est clairement d'insérer la politique budgétaire dans un cadre pluriannuel et sinon de contraindre, du moins d'informer les décisions en faisant référence à des objectifs et des principes stables dans le temps.

\section{Le cas français}

En France, la politique budgétaire reste marquée par la prédominance de l'annuel et du discrétionnaire. Les mesures internationales indiquent bien un changement en direction de l'adoption d'une règle budgétaire en 1998, au moment de l'adoption du premier programme de stabilité, où avait pour la première fois été fixée une norme pluriannuelle de progression de la dépense publique en volume. Cette norme de dépense a été complétée en 2004 par le principe selon lequel l'affectation des surplus de recette non-anticipés doit faire l'objet d'une décision ex ante. Mais l'application fait défaut.

En réalité, en effet, les principes ou règles qui sont supposés guider les décisions, notamment cette norme, constituent des engagements de faible portée ${ }^{12}$. Leur application sur un champ étroit - comme pour la norme de dépense en comptabilité budgétaire - est génératrice d'effets pervers puisqu'elle incite aux débudgétisations. Quant aux programmes triennaux de finances publiques ou programmes de stabilité, ils ont jusqu'ici eu le caractère d'une déclaration d'intentions. Ni les uns ni les autres n’ont été suivis d'effets depuis leur mise en place : la comparaison avec la Suède (Graphique 4) est à cet égard sans appel.

Les analyses ex post montrent que cette situation doit principalement au non-respect des objectifs de dépense et accessoirement seulement à une croissance économique moins forte qu'il n'était escompté. Si cette situation est commune puisqu'en Europe, sur la période 19982006, la dépense publique a excédé les prévisions dans trois-quarts des cas (Commission européenne, 2007), la France fait partie des pays où les dépassements ont été les plus fréquents et les plus importants (Moulin et Wierts, 2006).

Un rapport de l’Inspection Générale des Finances rappelle ainsi que la programmation pluriannuelle des finances publiques élaborée chaque année depuis 1998 n’a "jamais été respectée », ce qu'illustre le Tableau 1 . Il relève que cette programmation a souffert de " deux défauts majeurs : elle est réalisée dans la seule perspective du programme de stabilité et n’a pas de lien direct avec la procédure budgétaire annuelle ; elle ne s’appuie pas sur des cibles opérationnelles permettant de maîtriser effectivement les finances publiques » (Guillaume et al., 2007). En d'autres termes, la politique budgétaire n’a été réellement guidée ni par des principes ou des règles internes, ni par les engagements internationaux du pays.

\footnotetext{
${ }^{12}$ Le rapport 2007 du Conseil d’orientation des finances publiques indique que la gestion des finances publiques " s’insère dans un ensemble de règles » dont la gouvernance " a été améliorée au cours des années récentes ». Parmi ces règles il cite la « norme de dépenses » et la « règle d’affectation des surplus ». La première n’a pas de caractère législatif et constituent une simple déclaration d’intentions du gouvernement. La seconde est prévue par la LOLF, mais sous une forme imprécise : l’article 34-10 indique que la loi de finances annuelle « arrête les modalités selon lesquelles sont utilisés les éventuels surplus ».
} 
Graphique 4 : Prévisions et réalisations des programmes de stabilité : Suède et France

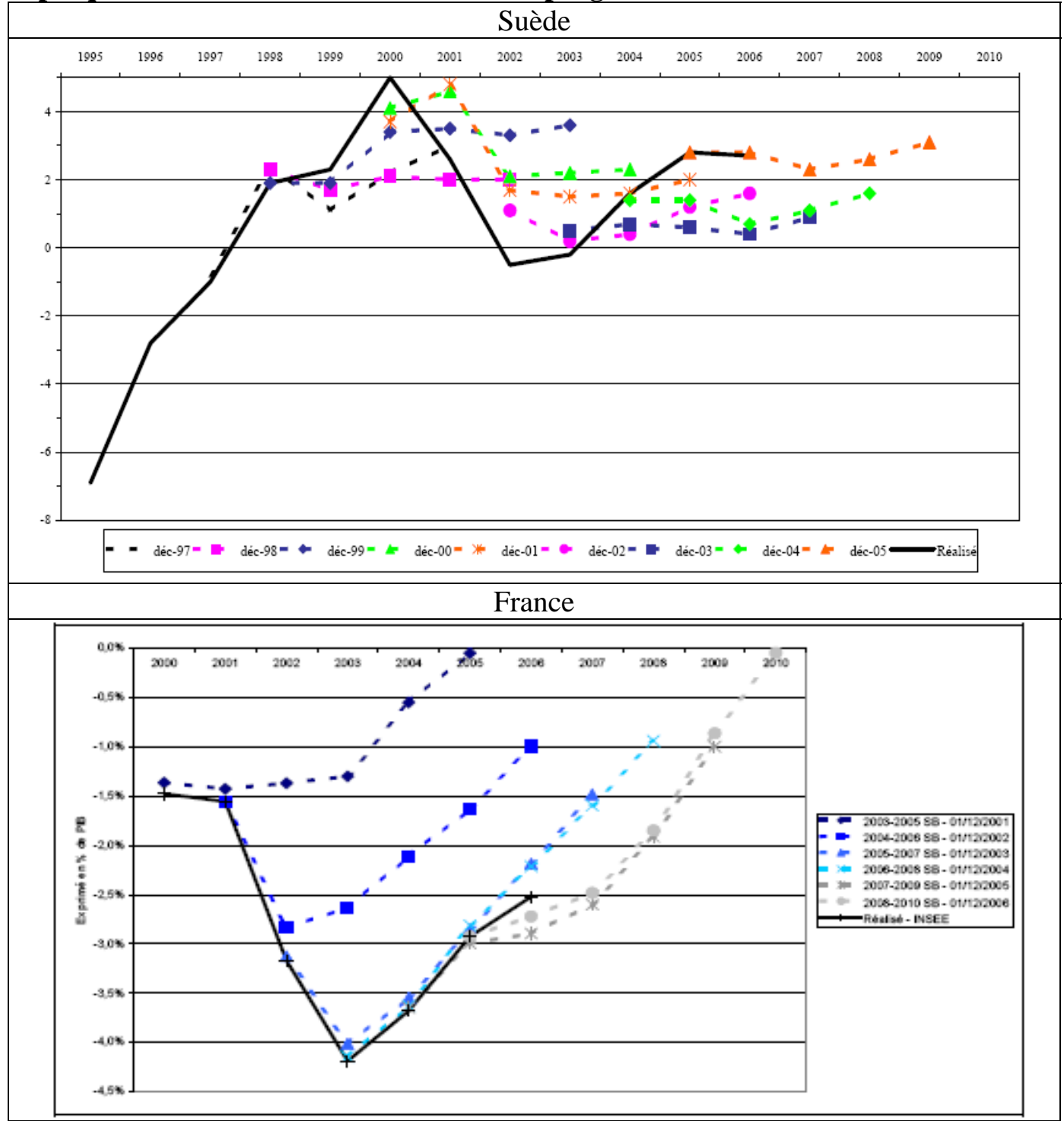

Source : Rapport Guillaume (2007)

Tableau 1 : Ecart entre objectifs et réalisations budgétaires à horizon de trois ans

Tableau 1: Evolution programmée et observée de la dépense publique en France

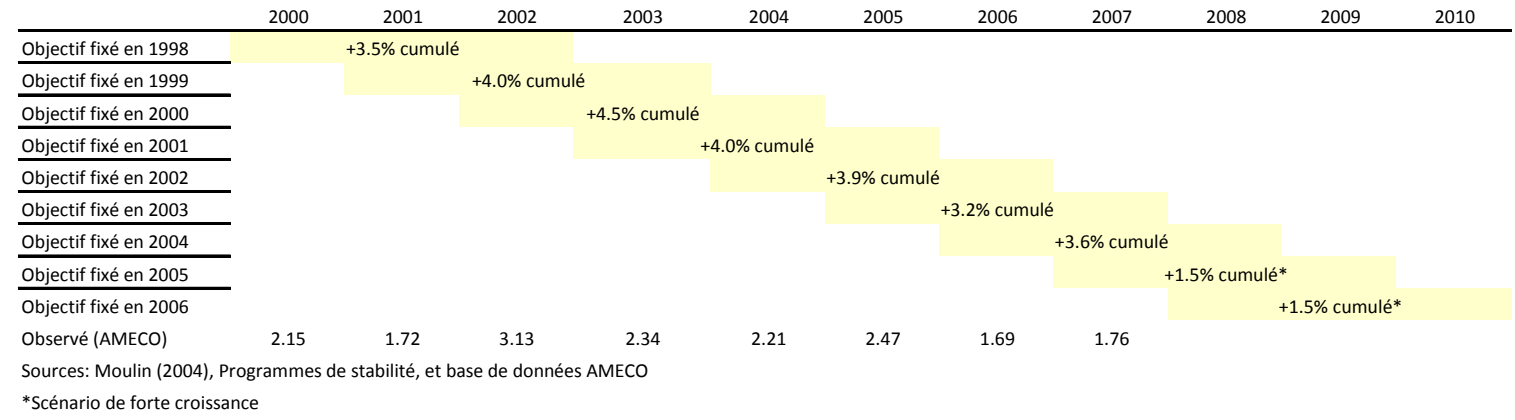


Pour autant, ces écarts n’ont pas favorisé une gestion active de la conjoncture. Toutes les évaluations concordent quant au caractère constamment pro-cyclique de la politique budgétaire française, avant et après l'union monétaire. Il n’y a eu sur la durée aucun arbitrage entre maîtrise de la dette et gestion du cycle, mais échec sur un plan comme sur l'autre : au contraire de ce qu'il aurait fallu, en moyenne la politique budgétaire a été expansionniste en période de bonne conjoncture et restrictive en période de mauvaise conjoncture (Graphique 5)

\section{Graphique 5 : Output gap et orientation de la politique budgétaire, 1992-2006}

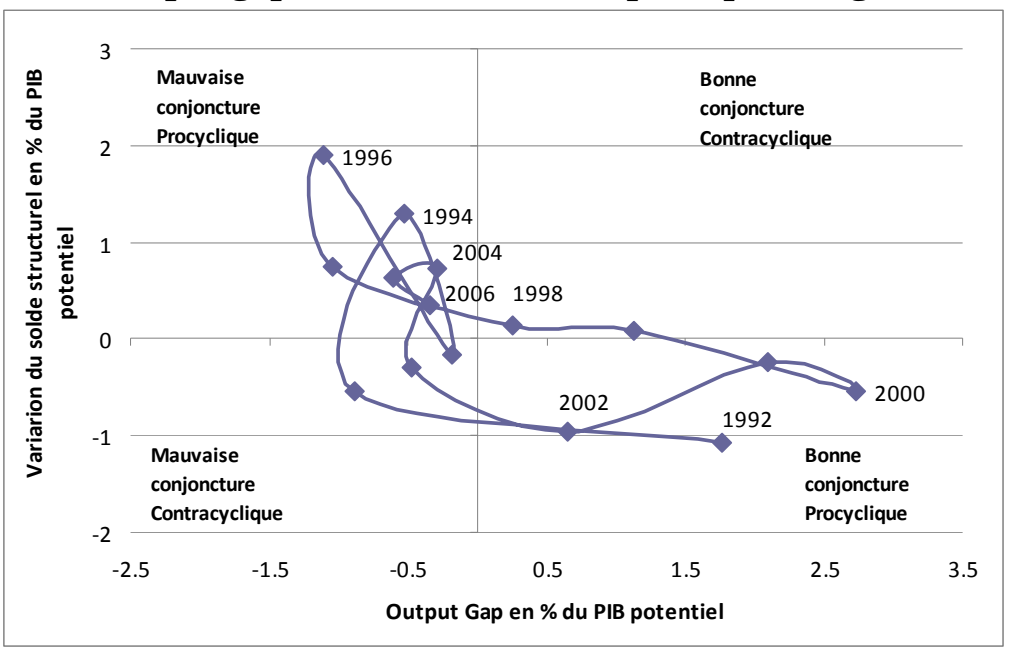

Source : OCDE.

Au total, on ne peut être que très sévère quant à la qualité de la gestion budgétaire française et au caractère superficiel des principes, des règles et des dispositifs institutionnels qui visent à lui donner un cadre de moyen terme. Pour autant, la réflexion sur ces principes, règles et institutions et sur la manière de leur donner force dans le contexte français reste embryonnaire. En dépit de sa tonalité alarmiste, le rapport Pébereau (2006) a négligé de la conduire et s'en est remis au volontarisme politique. La récente décision de donner, à partir du budget 2009, un cadre triennal aux projets de lois de finances soumis au parlement témoigne d'une prise de conscience bienvenue des limites de la gestion au jour le jour. Reste à voir si cette innovation suffira à rompre avec les comportements usuels.

\subsection{Gestion publique}

\section{L'expérience internationale}

Les données manquent pour recenser de manière comparative l'étendue des transformations que l'intervention régulatrice et la gestion publique ont connu au cours des dernières décennies dans les pays industriels, mais trois tendances apparaissent clairement.

La première est la généralisation du modèle de l'agence, d'abord en matière de politique de la concurrence et de régulation sectorielle, et de plus en plus dans la fourniture de services publics. Gilardi (2005) recense ainsi le développement des agences indépendantes dans les domaines de la concurrence et de la régulation des marchés financiers, de l'électricité et des télécoms. La proportion des pays industriels occidentaux disposant de telles agences était inférieure à $10 \%$ en 1960 et à 20\% en 1980, mais dépassait 90\% en 2000. Curieusement, l'exception majeure à cette tendance générale est aujourd’hui l’Union européenne, puisque la 
Commission conserve en son sein les responsabilités d'autorité de la concurrence et agit également comme régulateur, par exemple en matière de télécommunications.

Une deuxième tendance est la progression de la mesure, qu'il s'agisse d'évaluations ex post ou de l'usage d'indicateurs de performance pour la gestion publique. L'OCDE (2007) indique que parmi les pays recensés, $40 \%$ seulement disposaient d'indicateurs de performance il y a dix ans, mais que plus de $50 \%$ en ont introduit depuis dix ans. Cet effort de mesure, qui rompt avec la tradition comptable selon laquelle la production d'une administration est mesurée par ses inputs ${ }^{13}$, est un préalable nécessaire à une gestion par objectifs, il ne dit rien cependant sur l'utilisation qui est faite de ces indicateurs. L'OCDE indique que la plupart des gouvernements sont en la matière prudents, et ne déterminent presque jamais directement l'allocation des fonds budgétaires sur la base des indicateurs de performance. Ceux-ci semblent davantage utilisés comme éléments d’information dans une négociation plus large.

Enfin une troisième tendance est le recours croissant à des dispositifs incitatifs en matière de gestion publique: valorisation des inputs (services fournis par d'autres administrations, immobilier), management des services par objectifs, développement et sophistication de la rémunération au mérite. Les difficultés sont évidemment considérables en raison des spécificités de la gestion publique où l'output est mal défini et où les services accomplissent souvent plusieurs tâches simultanément. Si certains gouvernements sont très avancés dans cette voie (Nouvelle-Zélande, par exemple), d'autres restent plus prudents, mais le sens du mouvement est net.

\section{Le cas français}

En matière de régulation, la France a initialement tardé à mettre en place une autorité indépendante de la concurrence et ne lui a confié que des pouvoirs limités (le contrôle des concentrations continue de relever du ministre des Finances). Elle a souvent attendu, pour adopter le modèle de régulation par des agences sectorielles indépendantes, d'y être contrainte par Bruxelles. Cependant l'acclimatation de ce modèle a réussi et les modalités de l'intervention publique dans les principaux secteurs soumis à une régulation sectorielle ne diffèrent pas significativement de ce qu'elles sont dans d'autres pays européens (Tableau 2).

Tableau 2 : Date de création des agences de régulation indépendantes

\begin{tabular}{|l|r|r|r|}
\hline & Royaume-Uni & Allemagne & France \\
\hline Autorité de la concurrence & 1948 & 1957 & 1977 \\
\hline Régulation des télécoms & 1984 & 1996 & 1996 \\
\hline Régulation de l'énergie & 1989 & n.d. & 2000 \\
\hline
\end{tabular}

Source : Thatcher (2005)

En matière de gestion publique, la France accuse un retard important sur les pratiques internationales. Une série de rapports du Conseil d'Analyse Economique a ainsi mis en évidence la persistance d'une représentation du fonctionnement de l'Etat et d'un modèle d'organisation qui ignorent largement les apports de l'analyse économique contemporaine. Les travaux de Laffont (2000) sur la gestion publique, de Mougeot (1999) sur le système de santé, de Cohen et Mougeot (2001) sur l'usage des enchères, d'Aghion et Cohen (2004) sur l'enseignement supérieur ${ }^{14}$, ou de Bureau et Mougeot (2007) sur la performance et la gestion publique dressent un constat analogue: persistance du paradigme du planificateur bien

\footnotetext{
${ }^{13}$ Voir sur ce point le rapport Atkinson (2005) sur la mesure de la production des gouvernements en comptabilité nationale.

${ }^{14}$ Trannoy (2006) arrive à des conclusions analogues.
} 
informé, déficiences de l'évaluation, insuffisance de la mesure de la performance, absence d'incitations ou incitations perverses, retard dans la mise en œuvre des techniques actuelles de gestion publique.

Ces réflexions restent cependant peu entendues : certes, la Loi Organique relative aux Lois de Finance (LOLF) de 2001 vise à substituer une logique de résultat à une logique de moyens et marque une avancée de la contractualisation. Mais elle reste largement marquée par le paradigme du décideur informé, comme l'est aussi, et sans doute plus encore, la Révision Générale des Politiques Publiques (RGPP) de 2007. La démarche de modernisation de l'Etat n'intègre que marginalement les contraintes informationnelles et incitatives mises en évidence par l'analyse économique.

La situation est d'autant plus paradoxale que les gouvernants pourraient prendre appui sur les acquis d'une longue tradition d'économie publique, et sur l'excellence des économistes français dans deux domaines essentiels pour une réforme de la gestion publique : le design de mécanismes incitatifs et l'évaluation des politiques publiques par la microéconométrie.

\section{La singularité française : pourquoi ?}

Dans tous les pays, le délai entre une percée théorique et sa traduction dans la politique économique est long et variable, pour paraphraser Friedman. Même dans les plus avancés d'entre eux, il est possible de pointer des retards d'adaptation sur les avancées récentes de l'analyse. Mais en France ce délai est plus long et plus systématique qu'ailleurs. Dans les trois domaines-clef de l'action publique qui ont été examinés, le pays se distingue par une difficulté à s'approprier les avancées de l'analyse économique et à mettre en œuvre les nouvelles technologies de gouvernance. En matière monétaire, la question n'a été qu'apparemment résolue par la délégation de la responsabilité à une institution extérieure. Faute d'un effort crédible pour l'inscrire dans un cadre de moyen terme cohérent, la politique budgétaire est jusqu'ici demeurée le résultat d'une succession de décisions à courte portée, avec pour seul résultat tangible la dérive de l'endettement. En matière de gestion publique, le diagnostic n'est pas uniforme puisque la France s'est appropriée dans des domaines importants le modèle du régulateur indépendant, mais malgré l'innovation que constitue la LOLF elle accuse un retard notable dans l'adoption des méthodes modernes de gestion des administrations et des services publics.

Pourquoi en va-t-il ainsi ? Les raisons sectorielles peuvent ponctuellement avoir une certaine pertinence mais elles ne suffisent certainement pas à rendre compte de la réalité. On ne peut guère incriminer non plus le manque de ressources : non seulement l'administration française est particulièrement riche en capital humain, mais diverses médiations entre l'analyse économique et la décision politique ont été mises en place, qui fournissent autant de canaux d'intégration des apports de la recherche à la décision. A cet état de fait, il faut plutôt chercher des raisons intellectuelles, institutionnelles et politiques.

\section{Raisons intellectuelles}

Ce qui fait défaut, ce sont d'abord l'appropriation des avancées de l'analyse contemporaine et l'acceptation des disciplines intellectuelles et décisionnelles qu'elles impliquent. En France, l'action publique est sacralisée et la décision spontanément discrétionnaire, au nom du principe selon lequel l'intérêt général exige que le décideur ait toute latitude pour agir au 
mieux. Cette conception ne fait évidemment pas place à l'idée qu'une action efficace puisse appeler un détour ou l'acceptation d'une contrainte. Elle repose sur ce que Jean-Jacques Laffont (2000) appelait « une conception idéaliste du pouvoir politique et de la vie démocratique ».

Cette conception rend proprement incompréhensible que le décideur puisse devoir intégrer les limites de sa propre information, admettre le risque que son comportement réponde à une autre logique que celle de l'intérêt général, limiter le champ de sa décision, ou se lier les mains. C'est dans ce contexte qu'un premier ministre (Lionel Jospin, en 1999) a pu faire scandale en déclarant « l'Etat ne peut pas tout ». Pis, la leçon unanimement tirée de l'épisode a été qu'un dirigeant politique ne doit jamais évoquer les limites de son pouvoir, qu'il doit tout au contraire faire preuve à chaque instant d'un activisme sans faille, au risque de privilégier l'immédiateté sur l'efficacité de long terme.

Un facteur sociologique vient renforcer ces traits. Selon les pays la politique économique peut relever des politiques, des juristes ou des économistes. En France, elle est largement conçue et exécutée par des technocrates formés à une logique instrumentale au gré de laquelle chaque problème est justiciable d'une solution - d'une « mesure », pour employer le terme consacré , sans que soient pris en compte interdépendances entre domaines, comportements d'anticipation, imperfection de l'information, ou contraintes d'économie politique. Cette politique des mesures ne favorise pas une approche stratégique de la politique économique.

L'un des effets de cette représentation est l'absence persistante d'un cadre conceptuel de politique économique sinon unifié, du moins suffisamment cohérent pour permettre aux décideurs publics - à des niveaux variables - de situer leur action, d'organiser leur discussion sur les priorités, de sélectionner les instruments et de tirer les leçons de l’expérience.

La persistance d'une conception discrétionnaire et volontariste de la politique économique caractérise d'ailleurs les propositions françaises en matière de coordination internationale, au niveau européen comme à celui du G7, et explique une part des difficultés que rencontre la France à convaincre du bien-fondé de ses initiatives. Le scepticisme général des Européens à l'égard de la notion de "gouvernement économique » mise en avant par la France depuis la négociation de Maastricht ne s’explique pas seulement par le fait que celle-ci prône la coordination mais ne la pratique pas. Il résulte aussi de l'écart entre les conceptions françaises et la préférence de beaucoup de nos partenaires pour les règles.

Est-ce donc la faute à Rousseau ? S'en tenir là serait un peu court. Ce serait négliger une autre tradition intellectuelle qui a, de longue date, théorisé les limites du pouvoir discrétionnaire. Après tout lorsque Brissot, le chef des Girondins, déclarait en 1791 qu' « un pouvoir délégué sans un autre qui le surveille et le contrôle tend naturellement à violer le principe de sa délégation et à transformer cette délégation en souveraineté $"{ }^{15}$ il désignait en quelques mots ce que nous appelons aujourd'hui la relation principal-agent. Si la France s'est montrée rétive aux innovations de politique économique, c'est aussi pour des raisons institutionnelles et politiques.

\section{Raisons institutionnelles}

\footnotetext{
${ }^{15}$ Jacques-Pierre Brissot de Warville, Discours sur les conventions de 1791, cité par Rosanvallon (2000).
} 
Pour s'acclimater et prospérer, les innovations de politique économique n’ont pas seulement besoin d'un climat intellectuel, il leur faut aussi un terreau institutionnel. En matière de finances publiques, Hallerberg, Strauch et von Hagen (2007) on montré que les pays européens pouvaient être classés en deux catégories : ceux où la discipline budgétaire repose sur un contrat entre les partis participant au gouvernement; et ceux où elle repose sur la délégation du contrôle de la dépense à un ministre des Finances puissant. Dans les premiers, où le système politique repose généralement sur la représentation proportionnelle et les gouvernements sont constitués par des coalitions, la notion de règle budgétaire est une extension naturelle du contrat de gouvernement entre les partis de la coalition. Dans les seconds, où le système électoral est majoritaire et les gouvernements généralement unicolores, la notion de contrat n'a pas sa place et la règle s'acclimate mal. Cet exemple suggère ainsi une autre piste d'explications qui reposerait davantage sur les institutions que sur les représentations ou l’idéologie.

Il est clair qu'au contraire des Etats fédéraux, la diffraction du pouvoir n’est pas en France dans l'ordre des choses. Bien plutôt, l'organisation administrative repose sur l'unité d'un Etat envisagé comme " une machine sans friction », pour reprendre les termes de Laffont (2000). Par ailleurs le parlement, qui chez nos voisins s'est souvent saisi d'innovations procédurales ou institutionnelles pour surveiller ou affaiblir l'exécutif, a été trop faible, sous la $V^{\text {ième }}$ république, pour jouer ce rôle. Il n’a pas pu, à l'instar du Congrès américain, utiliser à son profit le développement des agences, ou comme plusieurs parlements européens fixer des règles budgétaires pour mieux contrôler la dépense publique. A cela s'ajoute le fait que le président de la République, qui (hors cohabitation) conserve à tout instant l'option de remplacer le premier ministre, a bien peu de raisons de se fixer des contraintes de cohérence temporelle alors que le système institutionnel lui offre un expédient plus confortable.

Le système institutionnel français est ainsi assez peu favorable à l'adoption des technologies modernes de gouvernance. Cependant cette explication ne peut non plus suffire. L'exemple du Royaume-Uni, pays traditionnellement tout aussi centralisé que la France, et qui a été pourtant à l'avant-garde des innovations, est là pour en montrer les limites.

\section{Raisons politiques}

Reste l'explication politique : les innovations dont il a été question dans cet article ne relèvent-elles pas à l'excès du " consensus de Washington ", ne sont-elles pas d'inspiration trop libérale pour être acceptables, ne vont-elles pas au total à l'encontre des préférences du pays ? Il est clair que la critique des politiques discrétionnaires, les premières théorisations de la vertu des règles, les propositions initiales du public choice ou le plaidoyer pour des banquiers centraux conservateurs sont nés d'un courant vigoureusement hostile à

l'intervention publique et attaché à mettre à bas les fondements intellectuels de l'édifice keynésien.

Cependant, outre qu'elle ne s'applique guère à l'ensemble des innovations, la lecture politique néglige l'évolution qui a conduit à l'intégration au mainstream d'idées initialement radicales et oublie que la mise en œuvre de ces innovations ait souvent été le fait de gouvernements d’inspiration sociale-démocrate, comme au Royaume-Uni ou en Suède.

S’il faut adopter une lecture politique, mieux vaut sans doute retenir celle de Ravi Kanbur (2001) dans son analyse de la « nature des désaccords » sur les politiques économiques. Si les innovations qui ont été recensées sont compatible avec des politiques d'orientations diverses, 
elles heurtent en revanche de plein fouet la préférence pour le présent et la focalisation sur les conflits de répartition qui caractérisent les discussions de politique économique françaises. Souligner la dimension intertemporelle des politiques publiques, et mettre en place les règles et institutions qui permettent de la préserver, imposer au décideur public de reconnaître les limites de sa propre volonté, et l'inviter au détour par souci d'efficacité, accepter que la décision doit être préservée de la tentation électoraliste ou partisane, et borner en conséquence l'espace du politique, c'est aller contre une représentation au gré de laquelle seule compte l'urgence de faire, et de le faire savoir.

Sans doute y-a-t-il là, à côté des explications intellectuelles ou institutionnelles, un facteur important du retard de nos institutions et de nos politiques. 


\section{Références}

Aghion, Philippe et Élie Cohen (2004), Éducation et croissance, rapport du CAE n6.

Akerlof, George (1970), “The Market for 'Lemons’: Quality Uncertainty and the Market Mechanism”, Quarterly Journal of Economics, vol. $84 \mathrm{n}^{\circ} 3$, pp. 488-500.

Alesina, Alberto, et Allan Drazen (1991), “Why Are Stabilizations Delayed?”, American Economic Review, Vol. 81, pp. 1170-88.

Alesina, Alberto, et Guido Tabellini (2007), "Bureaucrats or Politicians? Part I: A Single Policy Task,” American Economic Review, March 2007, 97: 169-79.

Arnone, Marco., Bernard Laurens, Jean-François Segalotto et Martin Sommer (2007), "Central Bank Autonomy: Lessons from Global Trends”, IMF Working Paper No. 07/88.

Arrow, Kenneth (1963), « Uncertainty and the Welfare Economics of Medical Care », American Economic Review vol 53 n5 pp. 941-973.

Atkinson, Tony (2005), Atkinson Review: Measurement of Government Output and Productivity for the National Accounts, Palgrave Macmillan.

Baron, David, et Roger Myerson (1982), “Regulating a Monopolist with Unknown Costs”, Econometrica, Vol. 50, No. 4, pp. 911-930.

Barro, Robert, et David Gordon (1983), "Rules, discretion and reputation in a model of monetary policy”, Journal of Monetary Economics, Volume 12 n²1, pp. 101-121.

Bénassy-Quéré, Agnès, Benoît Coeuré, Pierre Jacquet et Jean Pisani-Ferry (2004), Politique économique, De Boeck.

Bernanke, Ben (2003) “Constrained Discretion and Monetary Policy”, Speech at New York University, 3 February.

Bhundia, Ashok, et Gus O’Donnell (2002), “UK Policy Coordination: The Importance of Institutional Design”, Fiscal Studies vol. 23 n¹, pp. 135-164.

Blanchard, Olivier (2000), "What Do We Know about Macroeconomics that Fisher and Wicksell Did Not?”, The Quarterly Journal of Economics, Vol. 115, No. 4. (Novembre), pp. 1375-1409.

Blanchard, Olivier (2007), « Current Account Deficits in Rich Countries », NBER Working Paper n 12925, février.

Blinder, Alan (1997), "Is Government Too Political?", Foreign Affairs, vol. 76 n6, pp. 115-126.

Buchanan, James (1975), «A Contractarian Paradigm for Applying Economic Policy,» American Economic Review, 65 (2), pp. 225-230.

Bureau, Dominique, et Michel Mougeot (2007), Performance, incitations et gestion publique, rapport du CAE $n^{\circ}$ 66.

Cohen, Elie (2001), L'ordre économique mondial, Fayard.

Cohen, Elie, et Michel Mougeot (2001), Enchères et gestion publique, rapport du CAE n 34 .

Commission européenne (2007), Public Finance in EMU 2007.

Dixit, Avinash (1996), “The Making of Economic Policy”, Munich Lectures, The MIT Press.

Downs, Anthony (1957), An Economic Theory of Democracy, Harper.

Dyson, Kenneth, et Kevin Featherstone (1999), The Road to Maastricht, Oxford University Press.

Engel, Charles, et John Rogers (2006), “The U.S. current account deficit and the expected share of world output”, Journal of Monetary Economics vol. 53, pp. 1063-1093

Friedman, Milton (1968), “The Role of Monetary Policy”, The American Economic Review, Vol. 58, n 1, pp. 117.

Gilardi, Fabrizio (2005), “The Institutional Foundations of Regulatory Capitalism: The Diffusion of Independent Regulatory Agencies in Western Europe”, The Annals of the American Academy of Political and Social Science 598; 84. 
Guillaume, Henri, Arnaud Le Foll, Charlotte Reboul et Amélie Verdier (2007), Rapport sur la gestion pluriannuelle des finances publiques, Inspection générale des finances, avril.

Hallerberg, Mark, Strauch, Rolf, et Jürgen von Hagen (2007), “The design of fiscal rules and forms of governance in European Union countries,” European Journal of Political Economy, Elsevier, vol. 23(2), pages 338-359.

Henning, Randall (2007), “Organizing Foreign Exchange Intervention in the Euro Area”, Journal of Common Market Studies vol. 45 N², pp. 315-342.

Hurwicz, Leonid (1973), “The Design of Mechanisms for Resource Allocation”, The American Economic Review, Vol. 63, No. 2, pp. 1-30.

Kanbur, Ravi (2001), “Economic Policy, Distribution and Poverty: the Nature of Disagreements,” World Development, No. 29, pp.1083-1094.

King, Mervyn (2004), “The Institutions of Monetary Policy”, The American Economic Review, Vol. 94 n², pp. $1-13$.

Kydland, Fynn, et Edward Prescott (1977), "Rules Rather than Discretion: The Inconsistency of Optimal Plans”, The Journal of Political Economy, Vol. 85, No. 3, pp. 473-492.

Kopits, George, et Steven Symansky (1998), Fiscal Policy Rules, IMF Occasional Paper No. 162.

Laffont, Jean-Jacques, et Jean Tirole (1986), « Une théorie normative des contrats Etat-entreprises », Annales d'économie et de statistiques $\mathrm{n}^{\circ} 1$, janvier-mars.

Laffont, Jean-Jacques (2000), “Etapes vers un Etat moderne : une analyse économique”, in Etat et gestion publique, rapport du CAE $n^{\circ} 24$.

Laffont, Jean-Jacques, et David Martimort (2002), The Theory of Incentives : The Principal-Agent Model, Princeton University Press.

Maskin, Eric, et Jean Tirole (2004), « The Politician and the Judge : Accountability in Government », American Economic Review vol 94 n4, pp. 1034-1054.

Mirlees, James (1971), “An Exploration in the Theory of Optimal Income Taxation”, Review of Economic Studies vol. LXI, pp. 261-278.

Mougeot, Michel (1999), Régulation du système de santé, rapport du CAE n¹3.

Moulin, Laurent, et Peter Wierts (2006), “How Credible are Multiannual Budgetary Plans in the EU?”, Paper prepared for a Banca d’Italia workshop, juin.

Nordhaus, William (1975), “The Political Business Cycle”, Review of Economic Studies 42, pp. 169-190.

Obstfeld, Maurice, et Kenneth Rogoff (1996), Foundations of International Macroeconomics, The MIT Press.

OCDE (2007), “Towards Better Measurement of Government”, OECD Working Papers on Public Governance 2007/1, OECD Publishing. doi:10.1787/301575636734.

Pébereau, Michel (2006), Rompre avec la facilité de la dette publique, Rapport au ministre des Finances, La Documentation française.

Persson, Torsten, et Guido Tabellini (1999), « Political Economics and Macroeconomic Policy », in John Taylor et Michael Woodford (eds), Handbook of Macroeconomics, volume 1C, North-Holland.

Pisani-Ferry, Jean (2006), "Only One Bed for Two Dreams: A Critical Retrospective on the Debate over the Economic Governance of the Euro Area”, Journal of Common Market Studies vol. 44 n 4 .

Pisani-Ferry, Jean, Philippe Aghion, Alan Ahearne, Marek Belka, Lars Heikensten et André Sapir (2008), Coming of Age: Report on the euro area, Bruegel Blueprint Series n ${ }^{\circ} 4$.

Rosanvallon, Pierre (2000), La Démocratie inachevée. Histoire de la souveraineté du peuple en France, Gallimard, Bibliothèque des histoires.

Saint-Paul, Gilles (1993), « On the Political Economy of Labor Market Flexibility », NBER Macroeconomics Annual, The MIT Press, p. 151-195

Stiglitz, Joseph (2000), “The Contributions of the Economics of Information to Twentieth Century Economics”, Quarterly Journal of Economics vol. 115 n4, pp 1441-1478. 
Svensson, Lars (2007), "Optimal Inflation Targeting: Further Developments of Inflation Targeting," in Mishkin, Frederic, and Klaus Schmidt-Hebbel (eds.) (2007), Monetary Policy under Inflation Targeting, Banco Central de Chile, 187-225.

Tirole, Jean (1994), « The Internal Organization of Government », Oxford Economic Papers vol. 46 n 1.

Tirole, Jean (2002), Financial Crises, Liquidity and the Inetrnational Monetary System, Princeton University Press.

Trannoy, Alain (2006), "Financement des Universités, financement des études", Revue d'Economie Politique vol. $116 n^{\circ} 6$, pp. 745-782.

Vickrey, William (1961). "Counterspeculation and Competitive Sealed Tenders." Journal of Finance vol. 16 $\mathrm{N}^{\circ}$, pp. 8-37.

Walsh, Carl (1995), “Optimal Contracts for Central Bankers”, American Economic Review, vol. 85, n¹, pp. 150-167.

Wyplosz, Charles (2005), "Fiscal Policy: Institutions versus Rules, National Institute Economic Review n¹91, janvier. 